\title{
Effect of surface treatment to tensile static and creep properties for jute fiber reinforced composite
}

\author{
K. Takemura \\ Department of Mechanical Engineering, Kanagawa University, Japan
}

\begin{abstract}
Nowadays, there is a serious environmental problem with waste disposal etc. In fiber reinforced composite, glass fiber reinforced composite is difficult to reuse and recycle. Instead of glass fibers, natural fibers such as bamboo kenaf, jute, and hemp fibers are focused on for environmental friendliness. The mechanical properties for some natural fiber reinforced composites have been studied. The number of papers for green composite is greatly increased.

In this study, jute fibers are used as reinforcement because of the huge production and low cost. Polypropylene is used as a matrix because of recycling properties and cost. The strength and creep properties for Jute Fiber Reinforced Plastics (JFRP) are examined.

In Japan, natural fibers have been used as fishing nets for a long time. Astringency of a persimmon is used for hemp fiber as a reinforcement and binder. So, the surface treatment is conducted for the fiber using the astringency of a persimmon. The mechanical and creep properties for jute fiber reinforced composite and the effect of surface treatment are investigated.

From the results, the following conclusions are obtained.

The treatment using the solution of astringency of persimmon is effective to increase mechanical properties. In the case of using the treatment, some densities are effective and $25 \%$ solution is most effective. For the creep properties, there is an effect of surface treatment to creep strain. The effect is large when the load is small and the effect emerges in the initial stage.

Keywords: jute fiber, polypropylene, surface treatment, strength, creep.
\end{abstract}




\section{Introduction}

Fiber Reinforced Plastics (FRP) is widely used for airplane, automobile parts, fishing pole and any other engineering products. But, recently there are some environmental problems, especially the disposal of glass fibers could not be burned out. So, natural fibers such as jute, bamboo, hemp and flax are focused as reinforcement [1-5]. In Japan, third international workshop for green composite was held in 2005. Many researchers are interested in this issue.

Jute is focused from the viewpoint of cost. And it is easy to take jute from general market. So Jute Fiber Reinforced Plastics (JFRP) is used in this study. The static tensile tests for this composite are conducted in order to examine strength and stiffness etc. Natural fiber has been used for fishing net for a long time especially in Japan. Many chemical treatment methods have been used to the natural fibers. Among them, the astringency of a persimmon has been used for the improvement the face of natural fibers. So, the surface treatment method is conducted for this natural fiber using an astringency of a persimmon. So, mechanical and creep properties for the natural fiber reinforced composite and the effect for astringency of a persimmon are investigated.

\section{Specimen and experimental procedure}

\subsection{Specimen}

Plain woven jute fiber is used as reinforcement. The directions of jute fibers are parallel and perpendicular to a load direction. Polypropylene resin (Shin Kobe Electric Inc. PP-N-AN) is used as matrix. The geometry of specimens is referenced to JIS (Japanese Industrial Standard) 7054. The length of the specimen is about $200 \mathrm{~mm}$. The breadth and thickness are about $15 \mathrm{~mm}$ and $2.5 \mathrm{~mm}$ respectively. For surface treatment specimen, the plain woven fiber is laid in the solution of an astringency of a persimmon.

\subsection{Static tensile test for jute fiber}

Shimadzu autograph tensile test machine (AG-IS) is used for static tensile test. The test condition is referenced to JIS7054. The surfaces of the composite are observed using Scanning Electron Microscope (SEM) to confirm the effect of surface treatment.

\subsection{Creep test for jute fiber}

Creep Tester 100LER (Toyo Seiki. Co.) is used for this creep test. The test is continued by a failure of specimen. When a specimen does not fail, the creep test stops at 100 hours. Three tests can be conducted simultaneously. Creep extension and strain are measured. This test is referenced to JIS7087. The creep load is used for the consideration of static strength. 


\section{Results and discussion}

\subsection{Static test}

Static load-strain curve for jute fiber is shown in Fig.1. The 50\% solution is used for surface treatment. In this figure, the stiffness of treated specimen is lower than that of untreated one. But the maximum stress increases for the treatment. In the case of that using $100 \%$ concentration, the stiffness is not improved. So it is found that $50 \%$ diluted solution is effective for the strength. Because the fiber is not tensioned at the initial stage, the initial stiffness seems lower.

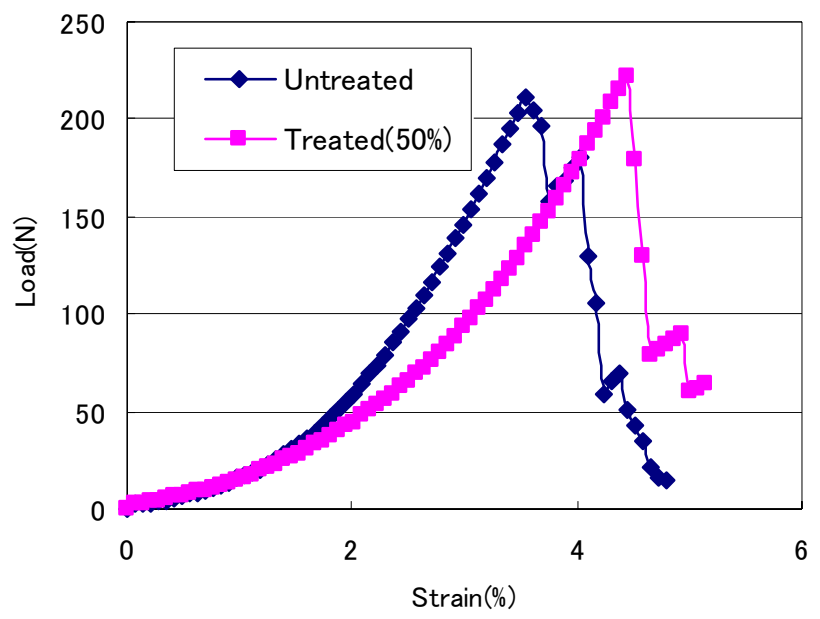

Figure 1: $\quad$ Static tensile results for jute fiber by surface treatment.

Figure 2 shows the stress-strain curves for jute fiber composite. The solution densities for surface treatment are $0,25,50,75,100 \%$. For the viewpoint of stiffness, $25 \%$ solution is most effective. For untreated specimen, the stiffness is lower than that of $25 \%$ specimen. But the strength is not low. For $100 \%$ solution, the strength and stiffness is worst. So it is understood that the treatment using an astringency of a persimmon is effective for the composite, but the densities are important. For $100 \%$ solution, it seems that the fiber has some damage on its surface. The knee point stress is important for its use as application. The knee point stresses for $25 \%$ and $50 \%$ are almost same. The knee point stresses for $0 \%$ and $75 \%$ are the same. The knee point stress for $100 \%$ solution is obviously lowest. From this figure, $50 \%$ solution may seem to be as effective as $25 \%$ solution.

The photograph of jute fiber is shown in Fig.3. From the surface of treatment, there is much resin on the surface. It is understood that the adhesive strength on the surface is improved. And, the fiber is not straight. It seems that the fiber has ductile properties. So, the stress-strain curve has knee point. And high stiffness is obtained from the surface. 


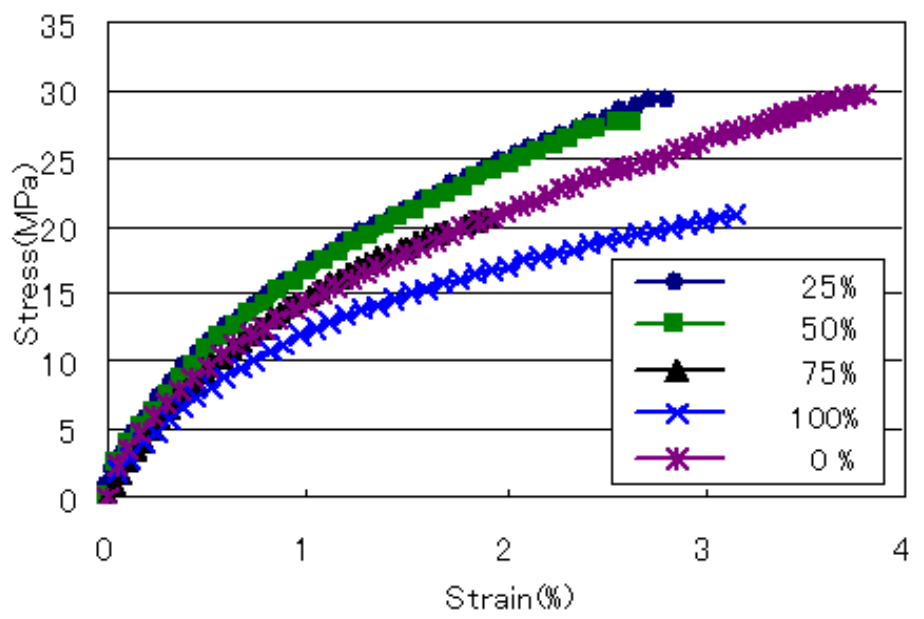

Figure 2: $\quad$ Static Stress-Strain curves of JFRP composite.

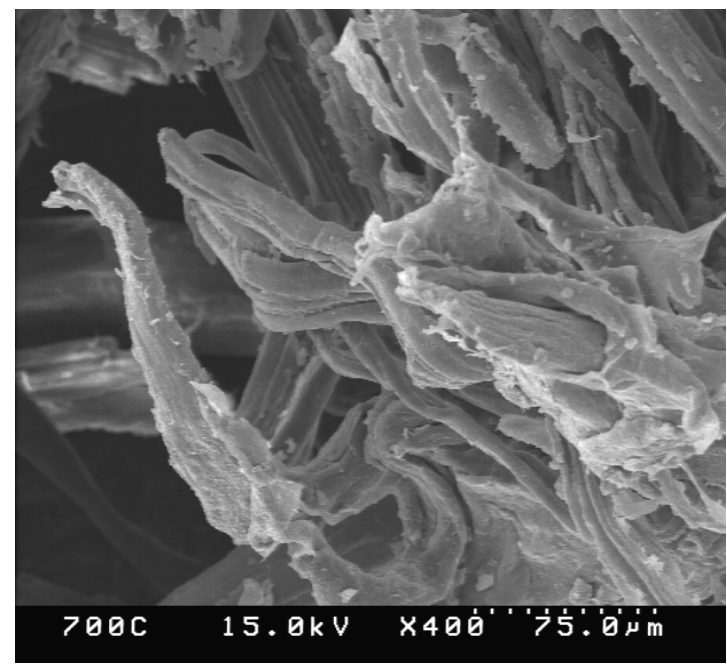

Figure 3: $\quad$ Fracture surface of jute fiber composite.

\subsection{Creep test}

Figure 4 shows the relationships between creep strain and time for untreated composite specimen. For smaller load, there seems to be little transit creep region. On the other hand, for bigger load, there is obviously transit creep region. The slopes of constant creep region seem to be same. So, the difference of the curve is emerged in transit region. The transit region continues by 15 hours. 


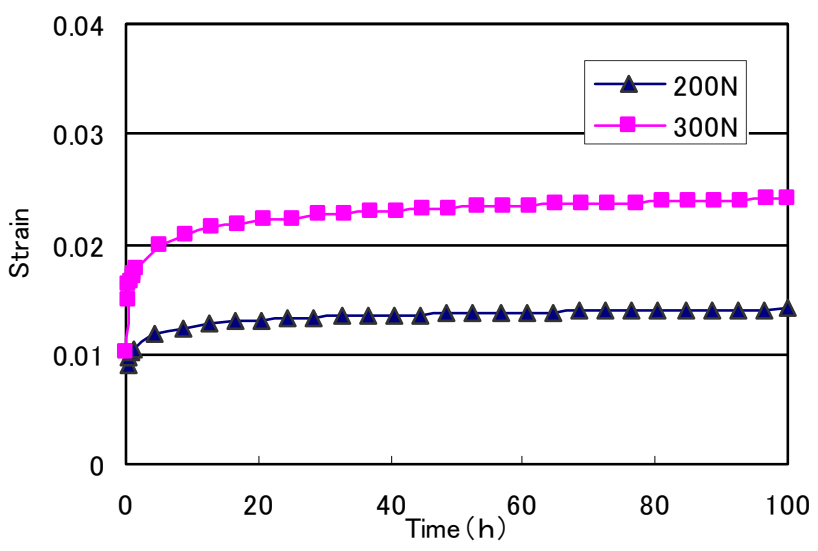

Figure 4: $\quad$ Creep curves for untreated JFRP.

Table 1 shows the creep strains for $25 \%$ treated and untreated composite specimen. From this table, it is understood that the effect of surface treatment is emerged at lower load. In the case of $300 \mathrm{~N}$, the creep strains seem to be same. From that result, there is obviously an effect of surface treatment. But when the load is bigger, the effect is hidden. In the case of $300 \mathrm{~N}$, the shapes of the curves are the same. On the other hand, in the case of $200 \mathrm{~N}$, the difference is emerging in the transit region. So, the effect of surface treatment is bigger at the initial stage for lower load. At the initial stage, there is the friction on the surface of jute fibre. So, it is thought that the effect of this region is greater.

Table 1: Comparison of creep strains for untreated and $25 \%$ treated jute fiber composite at the end of tests. (Unit \%).

\begin{tabular}{|c|c|c|}
\hline & Untreated & Treated \\
\hline $200 \mathrm{~N}$ & 1.4 & 1.2 \\
\hline $300 \mathrm{~N}$ & 2.4 & 2.4 \\
\hline
\end{tabular}

Because the effect of surface treatment is obtained in some conditions, the other treatment using katch is conducted. Katch is the obtained from the skin of tree. The result is shown in Fig.5. From this figure, it is understood that any effects are not obtained for $200 \mathrm{~N}$ case. For $300 \mathrm{~N}$ case, the strain is bigger. It is not understood that the katch is effective for these conditions. But in any other conditions, it may have any effects. There are any possible other treatment method, so some other methods have to be tried.

As the next stage, the surface treatment using water is conducted. Figure 6 shows the relationships between creep strain and time. For $200 \mathrm{~N}$, the creep strain is bigger than that of untreated specimen. So, it is not understood that the surface treatment using water is effective for creep properties. For the reason, an astringency of a persimmon has a role to smooth the surface of jute fiber. For only water, the effect of smoothing is not emerging. 


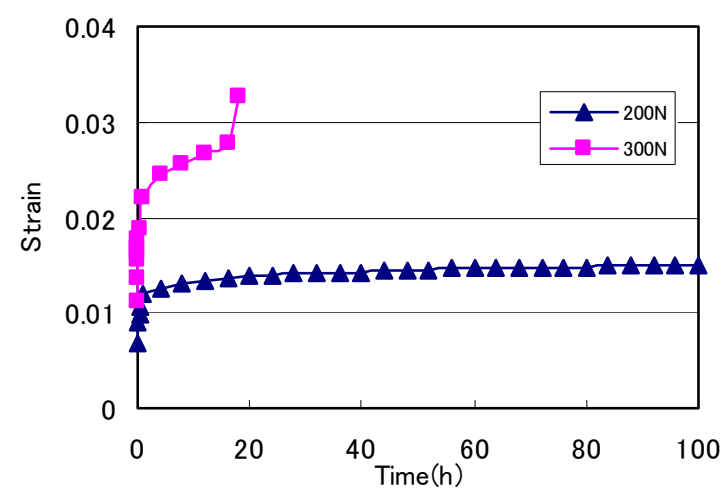

Figure 5: $\quad$ Creep curves of katch treated JFRP.

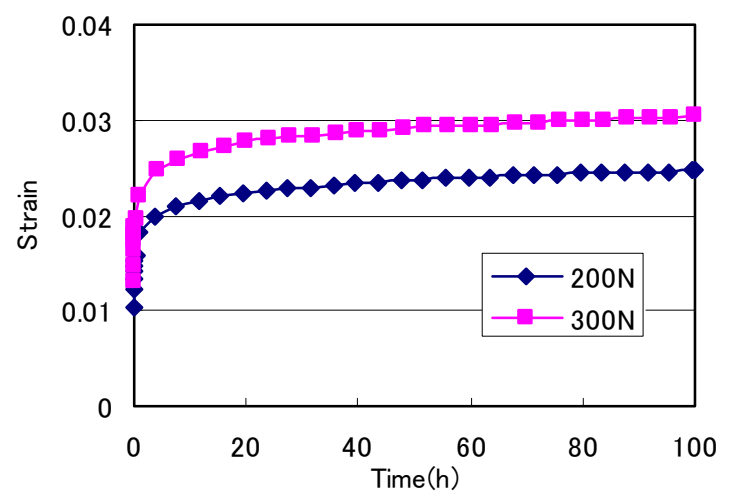

Figure 6: Creep curves of water treated JFRP.

The effect of an astringency of a persimmon seems to be tannin. So, the effect of tannin will have to be examined in detail.

\section{Conclusions}

In this study, the static and creep properties of jute fiber reinforced composite are examined and the effects of an astringency of a persimmon are also examined. As a result, following conclusions are obtained.

(1) There is an effect of astringency of a persimmon to static tensile properties.

(2) The effect is dependent on the solutions densities. When the density is $25 \%$, it is more effective.

(3) For creep properties, there is an effect of surface treatment to creep strain. The effect is big when the load is small. The effect emerges in the initial stage.

(4) The effect of surface treatment using only water is not obtained. On the other hand, in this case, the creep strain becomes big. 


\section{References}

[1] D. Nabi Saheb and J.P. Jog, Natural Fiber Polymer Composite: A Review, Advances in Polymer Technology, Vol.18, No.4, 351 (1999).

[2] H. Takagi and Y. Ichihara, JSME International Journal, Series A, 47,551 (2004).

[3] K. Goda et al., Proc. of the $2^{\text {nd }}$ Int. Workshop on "Green Composites", 96(2004).

[4] B. Singh et al., Composite Science and Technology, 60,571-589(2000).

[5] J. Gassan, at. al., Composite Science and Technology, 60,2857 (2000). 